Pure Appl. Chem., Vol. 76, No. 1, pp. 37-47, 2004.

(C) 2004 IUPAC

\title{
Ab initio QM/MM MD simulations of the hydrated $\mathrm{Ca}^{2+}$ ion*
}

\author{
Christian F. Schwenk and Bernd M. Rode \\ Department of Theoretical Chemistry, Institute of General, Inorganic and \\ Theoretical Chemistry, University of Innsbruck, Innrain 52a, A-6020 Innsbruck, \\ Austria
}

\begin{abstract}
The comparison of two different combined quantum mechanical (QM)/molecular mechanical (MM) simulations treating the quantum mechanical region at Hartree-Fock (HF) and B3-LYP density functional theory (DFT) level allowed us to determine structural and dynamical properties of the hydrated calcium ion. The structure is discussed in terms of radial distribution functions, coordination number distributions, and various angular distributions and the dynamical properties, as librations and vibrations, reorientational times and mean residence times were evaluated by means of velocity autocorrelation functions. The QM/MM molecular dynamics (MD) simulation results prove an eightfold-coordinated complex to be the dominant species, yielding average coordination numbers of 7.9 in the HF and 8.0 in the DFT case. Structural and dynamical results show higher rigidity of the hydrate complex using DFT. The high instability of calcium ion's hydration shell allows the observation of water-exchange processes between first and second hydration shell and shows that the mean lifetimes of water molecules in this first shell $(<100 \mathrm{ps})$ have been strongly overestimated by conclusions from experimental data.
\end{abstract}

\section{INTRODUCTION}

Understanding the solvation of calcium is of major importance due to the intriguing variety of biological events involving this ion [1-3]. Numerous experimental and theoretical reports [4-12] provide an inhomogeneous picture even for such rough structural data as the average number of coordinated water molecules. The preferred coordination number in crystal structures ranges from 6 to 8 [10]. Experimental data of the $\mathrm{Ca}^{2+}$ ion in solution range from a hydration number of 6 determined by $\mathrm{X}$-ray diffraction measurements using a $1.0 \mathrm{M} \mathrm{CaCl}_{2}$ solution [13] to a coordination number of 10 obtained from neutron diffraction experiments [8]. Various classical molecular dynamics simulations yielded coordination numbers between 7.9 and $9.3[4,5,7,14]$. Previous quantum mechanical (QM)/molecular mechanical (MM) molecular dynamics (MD) simulations showed a preference of the eightfold-coordinated square antiprismatic structure of the $\left[\mathrm{Ca}\left(\mathrm{H}_{2} \mathrm{O}\right)_{8}\right]^{2+}$ complex with average coordination numbers of 7.6 [12] and 8.3 [11] for QM/MM Hartree-Fock (HF) and 8.1 [12] for QM/MM density functional theory (DFT).

In addition to structural investigations, $\mathrm{QM} / \mathrm{MM} \mathrm{MD}$ simulations are suitable for describing very fast ligand exchanges taking place in the ps time scale. These ligand-exchange processes between coordination shells around metal ions in aqueous solution are fundamental for the reactivity of ions in chemical and biological systems [1,15-17]. Experimental investigations of such ultrafast ligand ex-

\footnotetext{
*Lecture presented at the European Molecular Liquids Group (EMLG) Annual Meeting on the Physical Chemistry of Liquids: Novel Approaches to the Structure, Dynamics of Liquids: Experiments, Theories, and Simulation, Rhodes, Greece, 7-15 September 2002. Other presentations are published in this issue, pp. 1-261.

ॠCorresponding author
} 
changes are scarce and could provide only rough estimations $[18,19]$. The mean lifetime for first-shell water exchange in $\mathrm{Ca}^{2+}$ has been postulated to be between $10^{-7}$ and $10^{-9} \mathrm{~s}$ based on experimental observations [15,17-19], whereas previous QM/MM MD simulations have shown exchange to occur much faster [20]. Recently, Car-Parinello-type simulations [21] of $\mathrm{Ca}^{2+}$ delivered a coordination number of 6 [22], which is a strong underestimation, similar to the predicted fivefold-coordinated $\mathrm{Cu}^{2+}$ ion [23], both being in contrast to all experimental results.

The evaluation of molecular motions, such as librational and vibrational motions, is another important subject of accurate QM/MM MD simulations. The velocity autocorrelation functions allow us to calculate these frequencies and to obtain detailed information about the molecular rotations, which is almost impossible by means of experimental methods.

In the present study, two different QM/MM MD simulations were performed—at HF and DFT level using the B3-LYP functional - to clarify all remaining inconsistencies concerning the hydration structure of the calcium ion. Additionally, further information about the water-exchange processes in the first hydration shell was collected to determine the exchange rate for calcium with high accuracy. The $\mathrm{Ca}^{2+}$-water vibrational frequencies are estimated according to the normal-coordinate analysis for the collective motions in the first hydration shell. The quantum mechanical treatment of the first hydration sphere in $\mathrm{QM} / \mathrm{MM}$ MD simulations yields a correct description of the hydration structure and is thus expected to allow a reliable estimation of the librational and vibrational frequencies.

\section{DETAILS OF CALCULATIONS}

\section{QM/MM MD methodology}

The QM/MM technique [24-26] partitions the system into a part described quantum mechanically, i.e., the first hydration shell, and another part treated by means of molecular mechanics. A smoothing function was applied between the two regions to ensure a smooth transition between QM and MM forces and energy conservation [27]. The radius of the region treated by means of the respective QM method was set to 3.8 in accordance with the radial distribution function (RDF) data from pair potential-based simulations. In each simulation step, an ab initio calculation was performed providing QM forces to be incorporated into the total force of the system by the following formula

$$
F_{\text {system,total }}=F_{\text {system,MM }}+\left(F_{\mathrm{QM}-\text { region, } \mathrm{QM}}-F_{\mathrm{QM}-\text { region, } \mathrm{MM}}\right) * S_{m}(r)
$$

where $F_{\text {system,total }}$ is the total force of the system, $F_{\text {system,MM }}$ is the MM force of the total system, $F_{\mathrm{QM}-\text { region,QM }}$ is the $\mathrm{QM}$ force in the $\mathrm{QM}$ region, and $F_{\mathrm{QM}-\text {-region, MM }}$ is the $\mathrm{MM}$ force in the $\mathrm{QM}$ region. $F_{\mathrm{QM}-\text { region,MM }}$ accounts for the coupling between $\mathrm{QM}$ and MM region.

All QM/MM simulations were carried out with the QM/MM MD software developed in our department [27] using the parallelized version of the TURBOMOLE package version 5.1 [28-30] for the calculation of the $a b$ initio forces. The basis sets used were nonrelativistic double zeta +1 polarization function (DZP) basis set for water [31] and Los Alamos effective core potential (ECP) plus nonrelativistic double zeta (DZ) basis set for $\mathrm{Ca}^{2+}$ [32]. The same basis sets have been employed for the construction of pair potential and three-body correction [12].

\section{QM/MM MD simulation}

The simulation protocol was the same as published recently [12] and very similar to numerous other QM/MM MD simulations [33-36]. 199 water molecules together with the calcium ion were immersed in a cubic box of 18.2 side length, and periodic boundary conditions were applied to the simulation box. The density in the canonical NVT ensemble was assumed to be $0.997 \mathrm{~g} / \mathrm{cm}^{3}$, and the simulation temperature of $298.16 \mathrm{~K}$ was kept constant by the Berendsen algorithm [37]. Water-water interactions were treated by the Bopp-Jancsó-Heinzinger (BJH) water model [38,39], which has proven to be suitable for 
the investigation of ions in aqueous solution [12,33-36,40]. The time step was set to $0.2 \mathrm{fs}$ since this water model allows explicit intramolecular hydrogen movements and the reaction-field procedure [41] was employed to correct the cutoff of long-range interactions.

The ab initio classical pair and three-body potential functions were taken from our previous work [12]. Two different QM/MM MD simulations were performed treating the quantum mechanical part at the HF level and with DFT (B3-LYP functional) using the equilibrium configuration obtained from a classical three-body corrected simulation. The QM/MM simulation at the HF level was equilibrated for $2 \mathrm{ps}$, and another $28 \mathrm{ps}$ were subsequently performed. The DFT/MM simulation was also equilibrated for $2 \mathrm{ps}$, and another 20 ps served for analysis.

\section{Velocity autocorrelation functions}

Dynamical properties of the water molecules in the liquid have been determined by computing the normalized velocity autocorrelation function (VACF), which is defined by

$$
C(t)=\frac{\sum_{i}^{N_{t}} \sum_{j}^{N} \vec{v}_{j}\left(t_{i}\right) \vec{v}_{j}\left(t_{i}+t\right)}{N_{t} N \sum_{i}^{N_{t}} \sum_{j}^{N} \vec{v}_{j}\left(t_{i}\right) \vec{v}_{j}\left(t_{i}\right)}=\frac{\left\langle\vec{v}_{j}(0) \vec{v}_{j}(t)\right\rangle}{\left\langle\vec{v}_{j}(0)^{2}\right\rangle}
$$

where $\vec{v}_{j}$ is the instantaneous velocity, $t$ is the correlation length, $N$ is the number of particles, $N_{t}$ the number of time origins $t_{i}$, and \langle\rangle denotes a time and ensemble average. The Fourier cosine transform of this function is evaluated numerically.

The vibrational and librational frequencies of water molecules were calculated using the normal coordinate analysis as reported previously $[42,43]$. After the subtraction of the center-of-mass velocity of the water molecules, the instantaneous velocities of hydrogen atoms were projected to the corresponding $\mathrm{O}-\mathrm{H}$ bond, perpendicular to the $\mathrm{O}-\mathrm{H}$ bond in the water plane, and perpendicular to the water plane. $\mathrm{Q}_{1}, \mathrm{Q}_{2}, \mathrm{Q}_{3}$ and $\mathrm{R}_{x}, \mathrm{R}_{y}, \mathrm{R}_{z}$ are defined to describe the symmetric stretching vibration, bending vibration, asymmetric stretching vibration, and rotations around the three principal axes of the water molecule, using the respective projection of the hydrogen velocities.

The frequencies of water molecules in the quantum mechanically treated first hydration shell obtained from QM/MM MD simulations were multiplied by a scaling factor of 0.89 in the HF and 0.96 in the DFT case $[44,45]$ because all atomic motions of these water molecules are generated according to the quantum theory-based forces calculated by the analytical gradient of the self-consistent field (SCF) wavefunctions.

\section{Mean residence times and reorientational times}

Mean residence times (MRTs) have been calculated as proposed by Impey et al. [46] using the following function

$$
n_{\text {ion }}(t)=\frac{1}{N_{t}} \sum_{n=1}^{N_{t}} \sum_{j} P_{j}\left(t_{n}, t ; t^{*}\right)
$$

where $N_{t}$ is the number of total time steps and $P_{j}\left(t_{n}, t ; t^{*}\right)$ is a binary sequence that answers yes or no ( 0 or 1$)$. If the water molecule $j$ lies within the coordination shell of the ion at both time steps $t_{n}$ and $t+t_{n}$ and does not leave the coordination shell for any continuous period longer than $t^{*}$ it takes the value 1 and under all other circumstances it takes the value $0 . n_{\text {ion }}(t)$ defines the number of water molecules that are initially in the first hydration sphere and remain there after a time $t . n_{\text {ion }}(0)$ is by definition the coordination number for $t=0$.

The $t^{*}$ parameter was introduced to take into account molecules that leave or enter the coordination shell only for a very short period $\left(<t^{*}\right)$ and return/leave again. It was set to $2 \mathrm{ps}$ in agreement with 
Impey et al. [46] $\left(t^{*}=2 \mathrm{ps}\right)$ and in contrast to Garcia et al. [47] analyzing biomolecule hydration with $t^{*}=0$ ps.

The reorientational time correlation functions (RTCFs) allow us to discuss orientational dynamics of the hydrated ions:

$$
C_{l i}(t)=\left\langle P_{l}\left(\vec{u}_{i}(0) \vec{u}_{i}(t)\right)\right\rangle
$$

$P_{l}$ is the Legendre polynomial of $l$ th order, and $\vec{\mu}_{i}$ is a unit vector along three principal axes $i$ defined in a fixed coordinate frame as the rotations above. We decided to fit the RCTFs to the simple formula

$$
C_{l}(t)=a \exp \left(-t / \tau_{1}\right)
$$

where $a$ and $\tau_{1}$ are the fitting parameters, and $\tau_{1}$ is the corresponding relaxation time, as both MRT and RCTFs, are assumed to decay exponentially.

\section{RESULTS AND DISCUSSION}

\section{Structural data}

All structural data for the comparison of the two QM/MM MD techniques are listed in Table 1. The $\mathrm{Ca}-\mathrm{O}$ and $\mathrm{Ca}-\mathrm{H}$ radial distribution functions (RDFs) with their running integration numbers for the two QM/MM MD simulations (HF and DFT) are plotted in Fig. 1. These RDFs show that the HF- and DFT-based QM/MM MD simulations produce similar structural results. The line shapes of the peaks of the first hydration shell of $\mathrm{Ca}^{2+}$ are very similar and the two peaks are situated at the same $\mathrm{Ca}-\mathrm{O}$ distance of $2.48 \AA$ in full agreement with previous results [12]. Previous differences between HF- and DFT-based QM/MM MD simulations decreased after expanding the simulation times from about 10 to $28 \mathrm{ps}$ in the HF case and form about 9 to $20 \mathrm{ps}$ in the DFT case. [12]. The comparison of the $\mathrm{Ca}-\mathrm{O}$ and

Table 1 Hydration structure parameters of $\mathrm{Ca}^{2+}$ in water determined by

\begin{tabular}{|c|c|c|}
\hline & QM/MM HF & QM/MM DFT \\
\hline$r_{1, \max }[\AA]^{\mathrm{a}}$ & 2.48 & 2.48 \\
\hline$r_{2, \max }^{1, \mathrm{~A}}[]^{\mathrm{b}}$ & 4.53 & 4.68 \\
\hline Average coordination number & 7.89 & 8.04 \\
\hline Mean $\mathrm{O}-\mathrm{M}^{2+}-\mathrm{O}$ angle $\left[^{\circ}\right]$ & $74 / 142$ & $74 / 140$ \\
\hline Height $^{c}$ & 3.0 & 2.85 \\
\hline $\mathrm{WHH}\left[{ }^{\circ}\right]^{\mathrm{d}}$ & 17 & 19 \\
\hline Mean $\theta$ angle $\left[{ }^{\circ}\right]^{\mathrm{e}}$ & 164 & 164 \\
\hline Height & 2.9 & 2.8 \\
\hline $\mathrm{WHH}\left[{ }^{\circ}\right]$ & 34 & 37 \\
\hline Mean oop angle $\left[{ }^{\circ}\right]^{\mathrm{f}}$ & 10 & 12 \\
\hline Height & 1.3 & 1.3 \\
\hline $\mathrm{WHH}\left[{ }^{\circ}\right]$ & 77 & 85 \\
\hline Mean $\alpha(\mathrm{O}-\mathrm{H}-\mathrm{O})\left[^{\circ}\right]^{\mathrm{g}}$ & $106.0(106.54)$ & $105.0(105.18)$ \\
\hline Mean $\alpha(\mathrm{O}-\mathrm{H})[\AA]$ & $0.9575(0.9473)$ & $0.9775(0.9688)$ \\
\hline
\end{tabular}
$\mathrm{QM} / \mathrm{MM}$ MD simulations (HF and DFT).

${ }^{\mathrm{a}}$ First peak maximum of $\mathrm{Ca}^{2+}-\mathrm{O}-\mathrm{RDF}$.

${ }^{\mathrm{b}}$ Second peak maximum of $\mathrm{Ca}^{2+}-\mathrm{O}-\mathrm{RDF}$.

${ }^{\mathrm{c}}$ Peak height of main peak.

${ }^{\mathrm{d}}$ Peak width at half-height of main peak.

${ }^{\mathrm{e}}$ Angle between ion-O and dipole vector.

${ }_{\mathrm{f}}^{\mathrm{A}}$ Angle between ion-O vector and water plane.

${ }^{g}$ Optimized values for single water molecules with the corresponding DZP basis set in parentheses. 

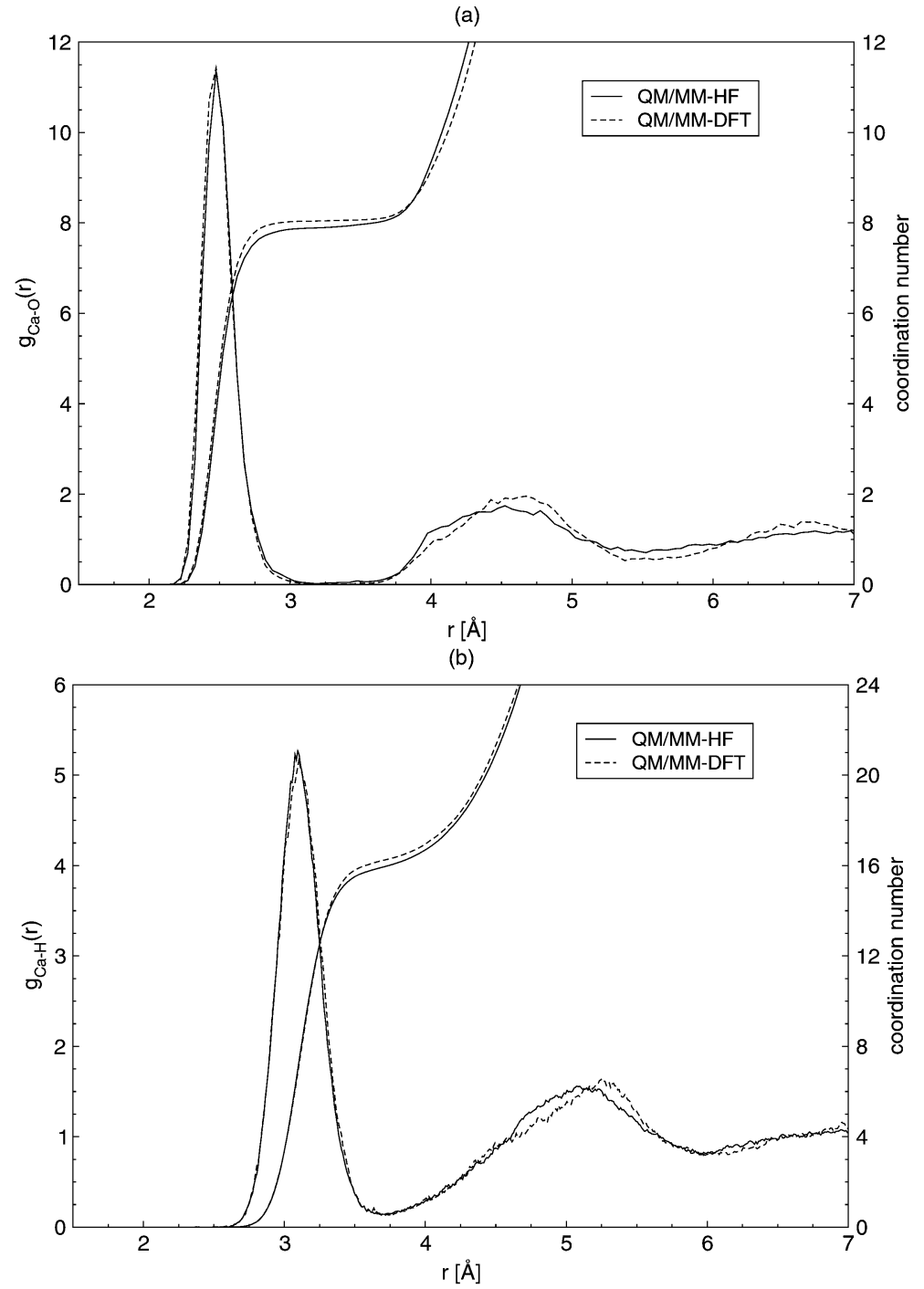

Fig. 1 Comparison of the radial distribution functions of the two QM/MM MD simulations at HF and DFT level: (a) Comparison of $\mathrm{Ca}-\mathrm{O}$ RDFs and their running integration numbers and (b) comparison of $\mathrm{Ca}-\mathrm{H}$ RDFs and their running integration numbers.

Ca-H RDFs outside the first shell shows a stronger structurization using DFT even pointing at a third hydration shell. The second peak maxima are situated at $4.53 \AA$ (HF) and $4.68 \AA$ (DFT) (Table 1) exhibiting a broader, less well defined peak at a shorter $\mathrm{Ca}-\mathrm{O}$ distance indicating higher flexibility in the HF case.

The coordination number distributions of the first shell show $92 \%$ of coordination number 8 in the DFT/MM and $82 \%$ of 8 in the HF/MM simulation (indicating again a more rigid structure in the DFT case), proving the $\left[\mathrm{Ca}\left(\mathrm{H}_{2} \mathrm{O}\right)_{8}\right]^{2+}$ complex to be the dominating species. The average coordination numbers of the second shell are 18.9 (HF) and 18.0 (DFT). Several angle distributions as tilt, $\theta$ and $\mathrm{O}-\mathrm{Ca}-\mathrm{O}$ angles show very similar behavior in both simulations. According to the nearly identical maxima of the $\mathrm{O}-\mathrm{Ca}-\mathrm{O}$ angle distribution of $74 / 142^{\circ}$ (HF) and $74 / 140^{\circ}$ (DFT) a square antisprismatic structure can be assumed. 
Tilt and $\theta$ angle allow us to investigate the position of a water molecule relative to the calcium ion. The maxima of the out-of-plane (tilt) angles of $10^{\circ}$ (HF) and $12^{\circ}$ (DFT) exhibit a preference of slightly tilted arrangements of the water molecules in the first hydration shell. The $\theta$ angle is defined as the angle between the $\mathrm{Ca}-\mathrm{O}$ connection vector and the vector instantaneously resulting from the sum of the $\mathrm{O}-\mathrm{H}$ vectors and shows a maximum of $164^{\circ}$ for both simulation techniques indicating a small deviation from dipole orientation.

Angle and bond length distributions of the water molecules depict slightly elongated bond lengths and contracted angles in comparison to the optimized gas-phase values in both cases and thus indicate a polarization of the water molecules by the calcium ion with respect to the gas-phase structure.

QM/MM MD simulations additionally allow us to determine the hydration energy of the ions $\left(\Delta E_{\text {hydr }}\right.$ ), which can be compared to experimentally evaluated enthalpies of hydration. Experimental hydration enthalpies measured for salts have to be assigned to separate anion and cation contributions. Literature values show significant variations, and therefore the data based on the TATB extrathermodynamic assumptions $[48,49]$ have been taken for a comparison. The resulting hydration energies are $-425 \mathrm{kcal} \mathrm{mol}^{-1}$ in the HF case and $-420 \mathrm{kcal} \mathrm{mol}^{-1}$ in the DFT case, whereas the experimental value amounts are $-383 \mathrm{kcal} \mathrm{mol}^{-1}$ [49]. In agreement with the nearly identical structural results of the two QM/MM MD simulations, the obtained hydration energies $\left(\Delta E_{\text {hydr }}\right)$ are also very similar exhibiting a deviation of $\sim 10 \%$ from experimental results. Since other references list experimental data with even higher stabilizations [50], our calculated energy values might be as reliable as experimental results.

\section{Dynamical data}

Due to the relatively short lifetime of a constant coordination number of 8 in the first hydration sphere of about $6 \mathrm{ps}$ in both QM/MM MD simulations, the dynamical properties have to be considered with special care. As the single modes can be separated and coincide with the total spectrum, the normal mode approximation [42] works very well. Only the frequencies corresponding to the power spectra of VACF of the first hydration shell are shown in Fig. 2 listed in Table 2, whereas second shell and bulk are not included.

The order $\mathrm{R}_{z}<\mathrm{R}_{x}<\mathrm{R}_{y}$ obtained for both QM/MM simulations of this work is in agreement with previous results $[52,53]$.

The comparison of the librational motions of HF and DFT simulation shows strongly blue-shifted values for the DFT/MM simulation for rotational motions around all three principal axes. The $R_{z}$ wavenumbers of water molecules in the first shell exhibit the largest difference of more than $20 \%$ with a strong blue-shift of the DFT result in contrast to a slightly red-shifted wavenumber in the HF case. Normally, the rotation around the $z$ axis $\left(\mathrm{R}_{z}\right)$ is not energetically restricted by the coordination of the metal ion as it is the rotation around the dipole axis, and the corresponding frequency shifts are very small. This general assumption is in good agreement with the QM/MM HF result but in contradiction to the strongly shifted $\mathrm{R}_{z}$ value of the DFT simulation where the rotation around the dipole axis is predicted to become energetically restricted due to the coordination of the calcium ion. The motions leading to a change in the dipole moment $\left(\mathrm{R}_{x}\right.$ and $\left.\mathrm{R}_{y}\right)$ are blue-shifted in both cases.

It is also possible to study the influence of the cation on the rotational relaxation times obtained from eq. 3. The values for RCTFs of first $\left(\tau_{1}\right)$ and second $\left(\tau_{2}\right)$ order for the two QM/MM simulations are summarized in Table 2. These correlation functions are related to infrared line shapes $(l=1)$ and Raman line shapes and NMR relaxation times $(l=2)$ [18]. The RCTFs show similar values for both simulations with slightly higher values resulting from the DFT technique in accordance with the higher rigidity resulting for the structure of the hydrate complex. In both cases, the highest relaxation times are found for rotations around the $z$ axis proving the rotation around the dipole axes to be the most probable movement besides translational motions. 
(a)
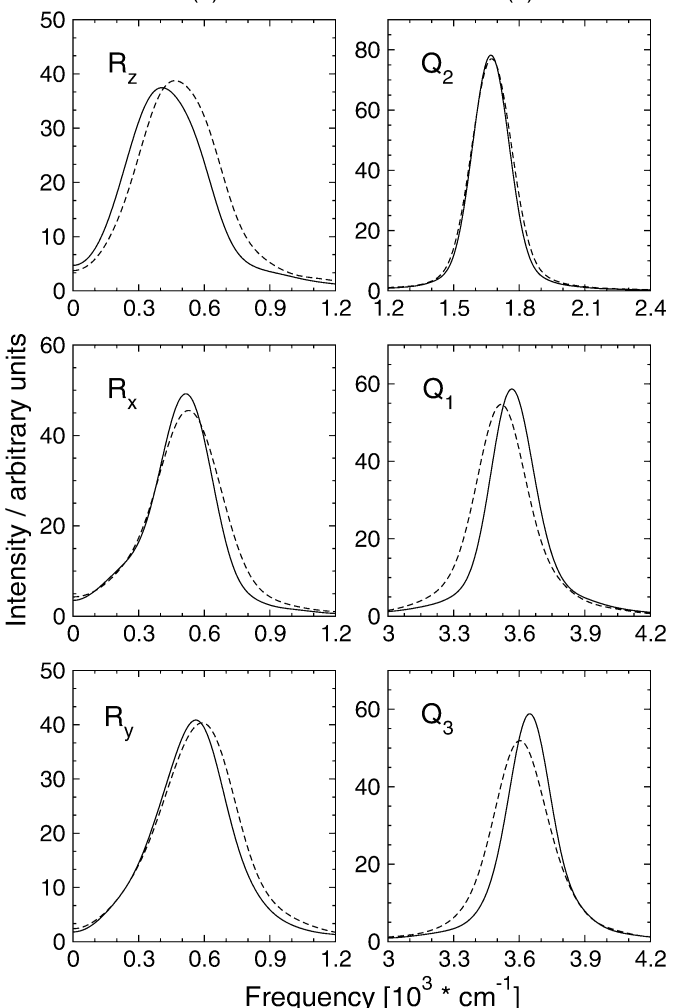

(b)
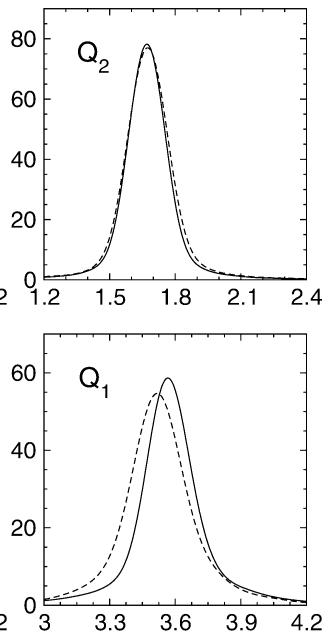

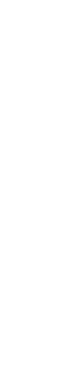

Fig. 2 Power spectra of: (a) rotational modes $\mathrm{R}_{z}, \mathrm{R}_{x}, \mathrm{R}_{y}$ and (b) vibrational modes $\mathrm{Q}_{2}, \mathrm{Q}_{1}, \mathrm{Q}_{3}$ for water molecules in the first hydration sphere of $\mathrm{Ca}^{2+}$ using QM/MM HF (solid line) and QM/MM DFT (dashed line).

The vibrational frequencies for $\mathrm{Q}_{2}$ (bending mode), $\mathrm{Q}_{1}$ (symmetric stretching mode), and $\mathrm{Q}_{3}$ (asymmetric stretching mode) are presented in Table 2. The bending mode $\mathrm{Q}_{2}$ is slightly red-shifted, in good agreement of both QM/MM simulations of the present work with a previous QM/MM simulation [11]. The asymmetric stretching motion exhibits a small blue-shift for the HF and a small red-shift for the DFT wavenumber with the result of the previous QM/MM simulation in between. The asymmetric stretching mode is blue-shifted in all cases.

The ion-oxygen motion may be separated as described above using the normal mode approximation. The values listed in Table 2 are $260 \mathrm{~cm}^{-1}$ for the QM/MM HF and $276 \mathrm{~cm}^{-1}$ for the QM/MM DFT simulation and the corresponding force constants are $46 \mathrm{Nm}^{-1}$ (HF) and $51 \mathrm{Nm}^{-1}$ (DFT), again indicating a stronger bond and higher rigidity in the DFT-based simulation.

The comparison of the coordination number distributions of the QM/MM-HF and DFT simulations prove the square antiprismatic $\left[\mathrm{Ca}\left(\mathrm{H}_{2} \mathrm{O}\right)_{8}\right]^{2+}$ complex to be the dominant but by no means exclusive species yielding average coordination numbers of 7.9 (HF) and 8.0 (DFT) for the first hydration sphere. Both QM/MM simulations started with this eightfold-coordinated complex. In the HF case, two exchange processes occurred during a simulation time of $28 \mathrm{ps}$, and another three short-lived attempts to start an exchange process took place. Both exchange processes are of dissociative character involving one single water in the first exchange taking place between 7.6 and $9.6 \mathrm{ps}$ and two different water molecules in the second one taking place between 24.9 and 26.1 ps (see Fig. 3). In the DFT/MM simulation, only one water-exchange process was observed during a simulation time of $20 \mathrm{ps}$. In contrast to the QM/MM HF simulation, a ninth water molecule entered the first hydration shell after 6.9 ps corresponding to an associative exchange mechanism without exerting much effect on the geometry of the 
Table 2 Vibrations, librations, ion-O stretching mode and reorientational times of firstshell and mean residence times of first- and second-shell waters of $\mathrm{Ca}^{2+}$. (Frequencies in $\mathrm{cm}^{-1}$ and times in ps.)

\begin{tabular}{lccccc}
\hline & QM/MM HF & QM/MM DFT & $\mathrm{H}_{2} \mathrm{O}(\mathrm{BJH})^{\mathrm{a}}$ & $\mathrm{H}_{2} \mathrm{O}(\exp .)^{\mathrm{b}}$ & $\mathrm{H}_{2} \mathrm{O}(\text { gas })^{\mathrm{c}}$ \\
\hline $\mathrm{R}_{z}^{\mathrm{d}}$ & 409 & 466 & 415 & & \\
$\mathrm{R}_{x}$ & 515 & 525 & 420 & & \\
$\mathrm{R}_{y}$ & 562 & 590 & 540 & & \\
$\mathrm{Q}_{2}{ }^{\mathrm{e}}$ & $1670(1650)^{\mathrm{f}}$ & 1676 & 1698 & 1645 & 1610 \\
$\mathrm{Q}_{1}$ & $3567(3465)$ & 3317 & 3455 & 3345 & 3650 \\
$\mathrm{Q}_{3}$ & $3646(3636)$ & 3602 & 3552 & 3445 & 3768 \\
$\mathrm{Q}_{i{ }^{\mathrm{g}}}$ & 260276 & & & & \\
$\tau_{1 x}$ & 5.0 & 6.3 & 4.8 & $7.5^{\mathrm{i}}$ & \\
$\tau_{1 y}$ & 4.0 & 5.0 & 5.7 & & \\
$\tau_{1 z}$ & 8.8 & 11 & 6.6 & & \\
$\tau_{2 x}$ & 2.2 & 3.8 & 2.6 & & \\
$\tau_{2 y}$ & 1.7 & 2.4 & 3.3 & & \\
$\tau_{2 z}$ & 3.0 & 3.8 & 2.9 & & \\
$\tau_{\mathrm{MPT}}$ & $\sim 40 / 4$ & $\sim 60 / 8$ & 3.0 & $2.5-10.0$ & \\
\hline
\end{tabular}

${ }^{\text {a} P u r e ~ l i q u i d ~ s i m u l a t i o n ~ w i t h ~ t h e ~ B J H ~ w a t e r ~ m o d e l ~[39] . ~}$

${ }^{\mathrm{b}}$ Experimental values for liquid water [51].

${ }^{\mathrm{c}} \mathrm{Scaled} \mathrm{HF}$ values.

${ }^{\mathrm{d}}$ Librations about the three principal axes $x, y, z$.

${ }^{\mathrm{e}}$ Bending, symmetric stretching, and asymmetric stretching mode.

${ }^{\mathrm{f}}$ Numbers in parentheses were obtained from a previous QM/MM HF simulation with Gaussian 94 [11].

${ }^{\mathrm{g}}$ Stretching mode of the ion-O motion.

${ }^{\mathrm{h}}$ Reorientational times about the three principal axes $x, y, z$ of first and second order, see eq. 5 .

${ }^{\mathrm{i}}$ Experimental reorientational correlation time of water [18].

${ }^{\mathrm{j}}$ Mean residence times of water molecules for first/second shell water molecules, eq. 3 .

eightfold-coordinated complex and leaving again after less than $1 \mathrm{ps}$. In agreement with structural and dynamical properties obtained, the DFT/MM simulation reflects higher rigidity. Thus, the $\left[\mathrm{Ca}\left(\mathrm{H}_{2} \mathrm{O}\right)_{8}\right]^{2+}$ complex is strongly stabilized with respect to either elimination or addition of a water ligand.

As experimental exchange rates are based on mean lifetimes of water molecules in the first shell, it is necessary to observe several exchange processes and to determine the average time span between them. Due to the low number of water exchanges observed in the present study, it is still not possible to calculate accurate exchange rate. QM/MM MD simulations of the required length are not yet feasible due to the enormous computational time required. Nevertheless, the QM/MM HF simulation exhibits two real exchanges and several additional attempts of water molecules to leave the first hydration shell. Consequently, it is possible to estimate the time scale of mean lifetimes of water molecules in the first shell to be in the 10-ps range $\left(\sim 10^{-11} \mathrm{~s}\right)$ in contrast to experimental estimations of a mean lifetime for first-shell water between $10^{-7}$ and $10^{-9} \mathrm{~s}$ [15-19]. The results of the QM/MM MD simulations show the water-exchange rates to be at least two orders of magnitude faster than assumed from experimental data.

The simulations also allow-beyond the capability of any experimental technique- the determination of the time span required for the ligand-exchange process itself. This time for the ligand movements between first and second hydration shell amounts $1.2 \mathrm{ps}$ in the DFT case (associative mechanism) and $\sim 1.6 \mathrm{ps}$ in average for an exchange process in the HF case. The calculated mean residence times for the first hydration shell are $~ 40 \mathrm{ps}$ for the QM/MM HF and $\sim 60 \mathrm{ps}$ for the QM/MM DFT simulation in agreement with the $\mathrm{Ca}-\mathrm{O}$ distance plot (Fig. 3) exhibiting two real exchanges and three short-lived at- 

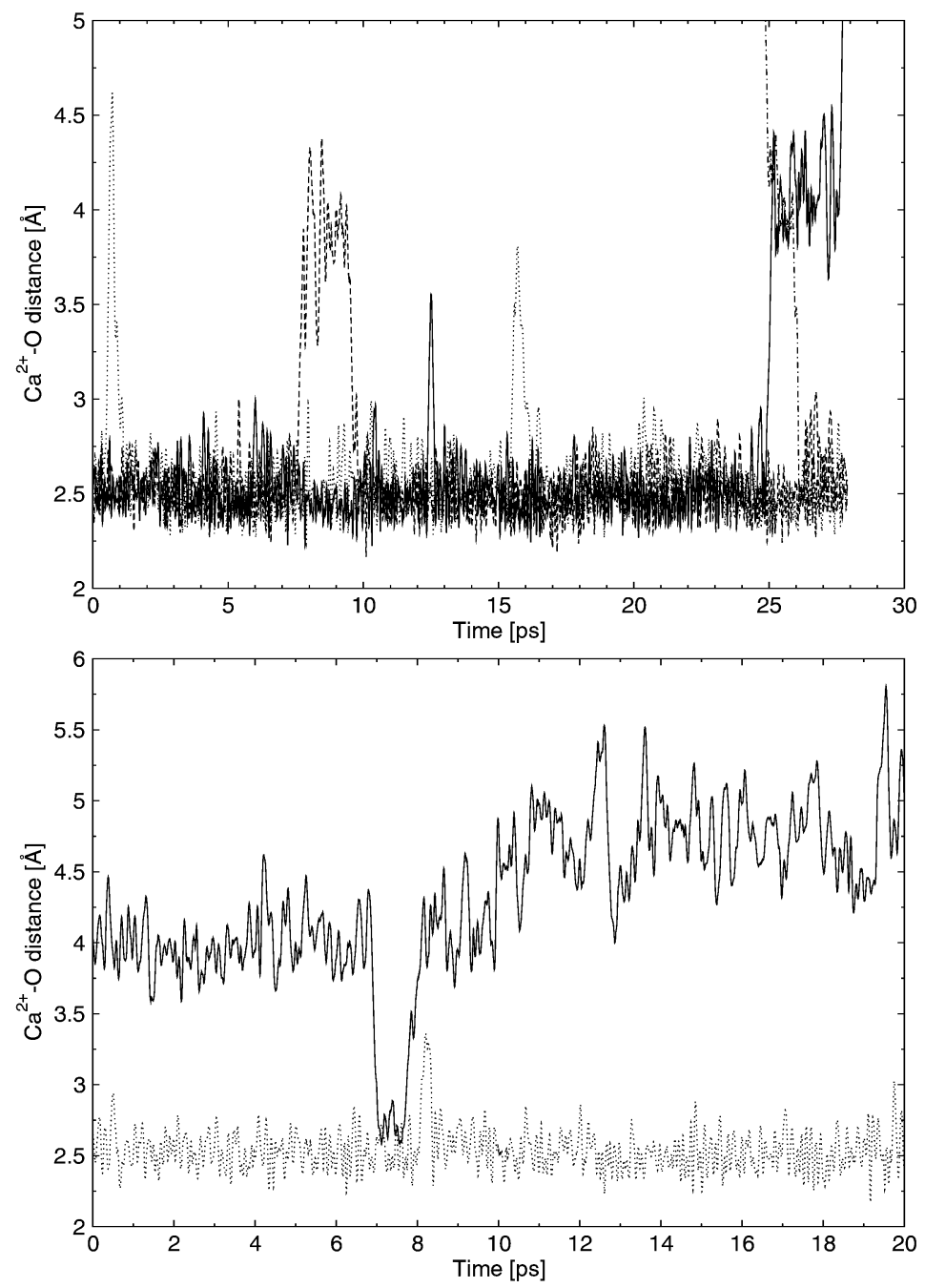

Fig. 3 Variation of the $\mathrm{Ca}-\mathrm{O}$ distances of the exchanging water molecule(s) during the QM/MM HF (upper figure) and QM/MM DFT (lower figure) simulation.

tempts for an exchange in the HF case and only one exchange in the DFT case. The first-shell mean residence time in the DFT simulation is 1.5 times larger, and the second-shell mean residence time is even 2 times larger than in the HF case (see Table 2). This is another proof for the higher flexibility of the QM/MM HF in comparison to the DFT/MM simulation and the corresponding overestimated rigidity using DFT.

\section{CONCLUSION}

QM/MM MD simulations treating the most crucial region of the first hydration shell by means of quantum mechanics are a suitable tool to describe structural and dynamical properties of calcium in aqueous solution. These accurate MD simulations prove the preference of an eightfold-coordinated calcium hydrate complex. The methodical comparison of HF and DFT techniques exhibits nearly equivalent structural results but significant differences in the dynamical behavior exhibiting a more rigid structure obtained with DFT. As no method offers a real time advantage over the other one, the QM/MM HF 
method appears favorable. QM/MM MD simulations provide a promising possibility to evaluate exchange rates for fast ligand-exchange processes difficult to measure experimentally. The mean lifetime of a water molecule in the first hydration shell was estimated to be in the 10-ps range, much faster than the experimentally assumed values and the corresponding exchange mechanism for first-shell water exchange is most probable of dissociative character.

\section{ACKNOWLEDGMENTS}

Financial support for this work from the Austrian Science Foundation (FWF) is gratefully acknowledged (project P13644-TPH).

\section{REFERENCES}

1. D. T. Richens. The Chemistry of Aqua Ions: Synthesis, Structure and Reactivity: A Tour Through the Periodic Table of the Elements, $1^{\text {st }}$ ed., Wiley, New York (1997).

2. D. Clapham. Cell 80, 259 (1995).

3. W. Chazin. Nat. Struct. Biol. 2, 707 (1995).

4. D. G. Bounds. Mol. Phys. 54, 1335 (1985).

5. M. M. Probst, T. Radnai, K. Heinzinger, P. Bopp, B. M. Rode. J. Phys. Chem. 89, 753 (1985).

6. G. Pálinkás and K. Heinzinger. Chem. Phys. Lett. 126, 251 (1986).

7. S. Obst and H. Bradaczek. J. Phys. Chem. 100, 15677 (1996).

8. N. A. Hewish, G. W. Neilson, J. E. Enderby. Nature 138, 297 (1982).

9. G. W. Neilson and J. E. Enderby. Advances in Inorganic Chemistry Vol. 34, pp. 195-218, Academic, San Diego (1989).

10. A. K. Katz, J. P. Glusker, S. A. Beebe, C. W. Bock. J. Am. Chem. Soc. 118, 5752 (1996).

11. A. Tongraar, K. R. Liedl, B. M. Rode. J. Phys. Chem. A 101, 6299 (1997).

12. C. F. Schwenk, H. H. Loeffler, B. M. Rode. J. Chem. Phys. 115, 10808 (2001).

13. G. Licheri, G. Piccaluga, G. Pinna. J. Chem. Phys. 64, 2437 (1976).

14. S. G. Kalko and G. Sese. J. Chem. Phys. 104, 9578 (1996).

15. J. Burgess. In Ions in Solution: Basis Principles of Chemical Interactions, U. o. L. J. Burgess (Ed.), Department of Chemistry, Ellis Horwood Series in Inorganic Chemistry, Leicester, UK (1988).

16. J. J. R. F. da Silva and R. J. P. Williams. The Biological Chemistry of the Elements: The Inorganic Chemistry of Life, Oxford University Press, New York (1991).

17. S. F. Lincoln and A. Merbach. Advances in Inorganic Chemistry, Vol. 42, pp. 1-88, Academic, San Diego (1995).

18. H. Ohtaki and T. Radnai. Chem. Rev. 93, 1157 (1993).

19. L. Helm and A. E. Merbach. Coord. Chem. Rev. 187, 151 (1999).

20. C. F. Schwenk, H. H. Loeffler, B. M. Rode. Chem. Phys. Lett. 349, 99 (2001).

21. R. Car and M. Parinello. Phys. Rev. Lett. 55, 2471 (1985).

22. J. Samios (Ed.). Novel Approaches to the Dynamics of Liquids: Experiments, Theories and Simulations, Advanced Study Institute, Rhodes (2002).

23. A. Pasquarello, I. Petri, P. S. Salmon, O. Parisel, R. Car, É. Tóth, D. H. Powell, H. E. Fischer, L. Helm, A. Merbach. Science 291, 856 (2001).

24. M. J. Field, P. A. Bash, M. Karplus. J. Comput. Chem. 11, 700 (1990).

25. J. Gao. In Reviews in Computational Chemistry, Vol. 7, K. B. Lipkowitz and D. B. Boyd (Eds.), Chap. 3, pp. 119-185, VCH, New York (1996).

26. D. Bakowies and W. Thiel. J. Phys. Chem. 100, 10580 (1996).

27. T. Kerdcharoen, K. R. Liedl, B. M. Rode. Chem. Phys. 211, 313 (1996).

28. R. Ahlrichs, M. Bär, M. Häser, H. Horn, C. Kölmel. Chem. Phys. Lett. 162, 165 (1989). 
29. R. Ahlrichs and M. von Arnim. In Methods and Techniques in Computational Chemistry: METECC-95, E. Clementi and G. Corongiu (Eds.), Chap. 13, pp. 509-554, STEF, Cagliari (1995).

30. M. von Arnim and R. Ahlrichs. J. Comput. Chem. 19, 1746 (1998).

31. T. H. Dunning, Jr. J. Chem. Phys. 53, 2823 (1970).

32. W. R. Wadt and P. J. Hay. J. Chem. Phys. 82, 284 (1985).

33. Y. Inada, A. M. Mohammed, H. H. Loeffler, B. M. Rode. J. Phys. Chem. A 106, 6783 (2002).

34. H. H. Loeffler, J. I. Yagüe, B. M. Rode. Chem. Phys. Lett. 363, 367 (2002).

35. H. H. Loeffler and B. M. Rode. J. Chem. Phys. 117, 110 (2002).

36. J. I. Yagüe, A. M. Mohammed, H. Loeffler, B. M. Rode. J. Phys. Chem. A 105, 7646 (2001).

37. H. J. C. Berendsen, J. P. M. Postma, W. F. van Gunsteren, A. DiNola, J. R. Haak. J. Phys. Chem. 81, 3684 (1984).

38. F. H. Stillinger and A. Rahman. J. Chem. Phys. 68, 666 (1978).

39. P. Bopp, G. Janscó, K. Heinzinger. Chem. Phys. Lett. 98, 129 (1983).

40. A. Tongraar, K. R. Liedl, B. M. Rode. J. Phys. Chem. A 102, 10340 (1998).

41. D. J. Adams, E. M. Adams, G. J. Hills. Mol. Phys. 38, 387 (1979).

42. P. Bopp. Chem. Phys. 106, (1986).

43. E. Spohr, G. Pálinkás, K. Heinzinger, P. Bopp, M. M. Probst. J. Phys. Chem. 92, 6754 (1988).

44. A. P. Scott and L. Radom. J. Phys. Chem. 100, 16502 (1996).

45. D. J. DeFrees and A. D. McLean. J. Chem. Phys. 82, 333 (1985).

46. R. W. Impey, P. A. Madden, I. R. McDonald. J. Phys. Chem. 87, 5071 (1983).

47. A. E. García and L. Stiller. J. Comput. Chem. 14, 1396 (1993).

48. Y. Marcus. J. Chem. Soc., Faraday Trans. 87, 2995 (1991).

49. Y. Marcus. Pure Appl. Chem. 59, 1093 (1987).

50. M. C. Day and J. Selbin. Theoretical Inorganic Chemistry, Reinhold, New York (1962).

51. W. F. Murphy and H. J. Bernstein. J. Phys. Chem. 76, 1147 (1972).

52. P. Bopp. Pure Appl. Chem. 59, 1071 (1987).

53. Y. Inada, H. H. Loeffler, B. M. Rode. Chem. Phys. Lett. 358, 449 (2002). 